\title{
Experimental Demonstration of Multidimensional Switching Nodes for All-Optical Data Centre Networks
}

Kamchevska, Valerija; Medhin, Ashenafi Kiros; Da Ros, Francesco; Ye, Feihong; Asif, Rameez; Fagertun, Anna Manolova; Ruepp, Sarah Renée; Berger, Michael Stübert; Dittmann, Lars; Morioka, Toshio

Total number of authors:

12

Publication date:

2015

Document Version

Peer reviewed version

Link back to DTU Orbit

Citation (APA):

Kamchevska, V., Medhin, A. K., Da Ros, F., Ye, F., Asif, R., Fagertun, A. M., Ruepp, S. R., Berger, M. S.,

Dittmann, L., Morioka, T., Oxenløwe, L. K., \& Galili, M. (2015). Experimental Demonstration of Multidimensional Switching Nodes for All-Optical Data Centre Networks. Paper presented at 41st European Conference and Exhibition on Optical Communications, Valencia, Spain.

\section{General rights}

Copyright and moral rights for the publications made accessible in the public portal are retained by the authors and/or other copyright owners and it is a condition of accessing publications that users recognise and abide by the legal requirements associated with these rights.

- Users may download and print one copy of any publication from the public portal for the purpose of private study or research.

- You may not further distribute the material or use it for any profit-making activity or commercial gain

- You may freely distribute the URL identifying the publication in the public portal 


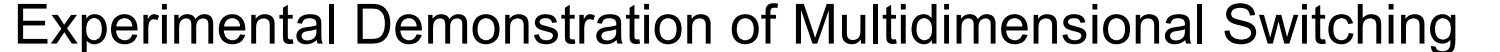

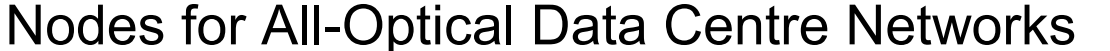

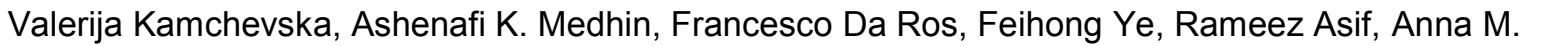

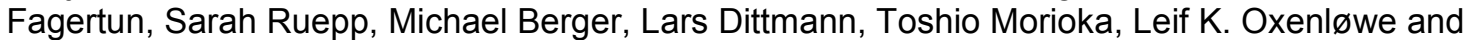

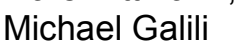

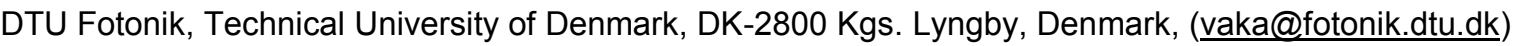

Abstract $\square$ We experimentally demonstrate network nodes that enable SDM/WDM/TDM switching. $1 \mathrm{Tbit} / \mathrm{s} / \mathrm{core}$ error-free performance is achieved for connections with different granularities being switched between three network nodes interconnected with 7-core multicore fibres.

\section{Introduction}

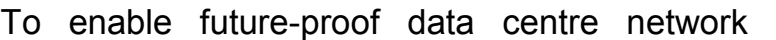

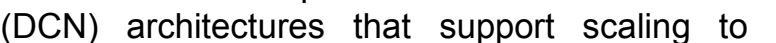

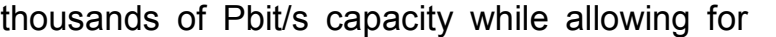

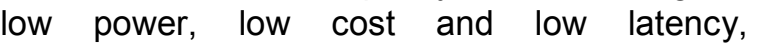

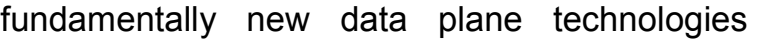

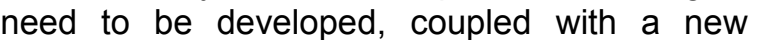

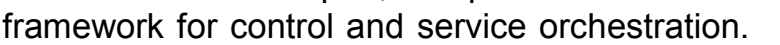

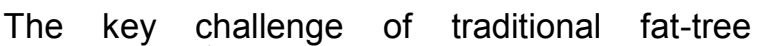

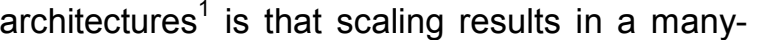
प

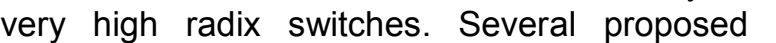

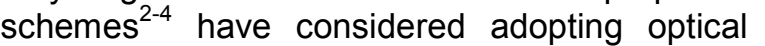

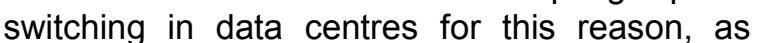

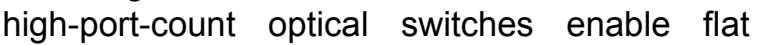

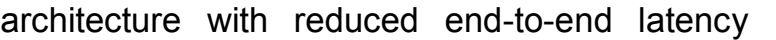

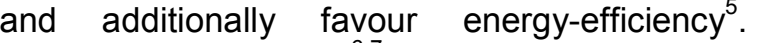

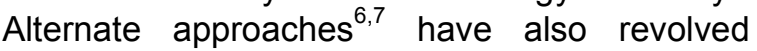

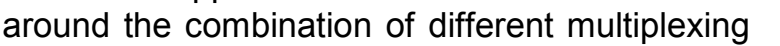

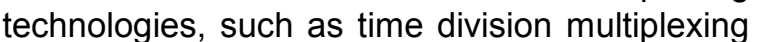

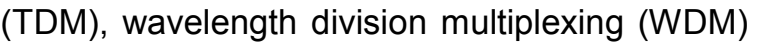

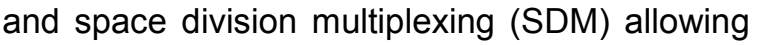

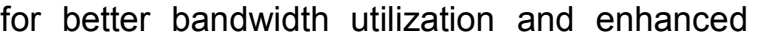

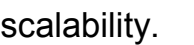

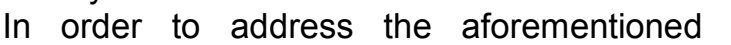

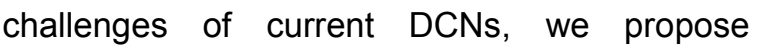

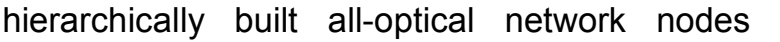

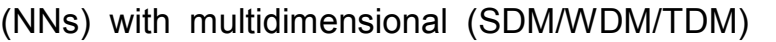

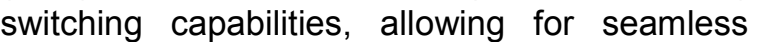

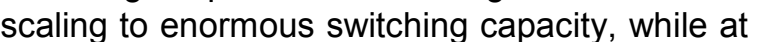

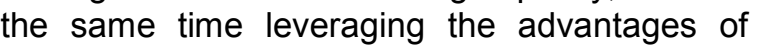

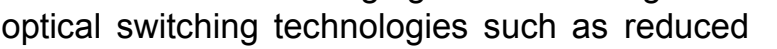

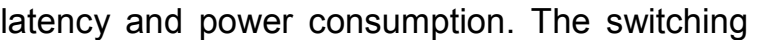

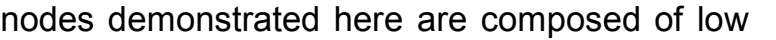

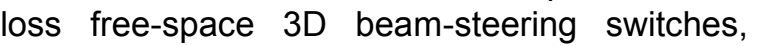

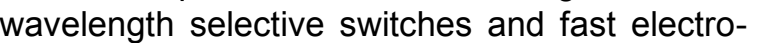

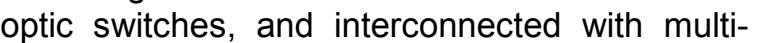

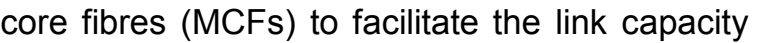

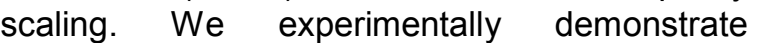

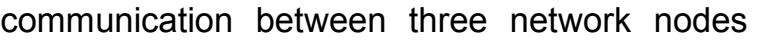

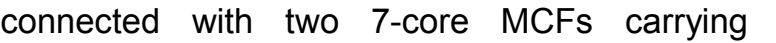

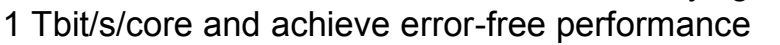
पाता

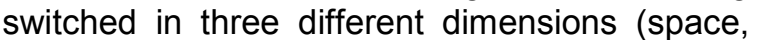

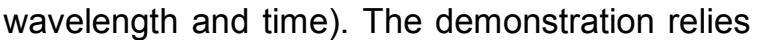

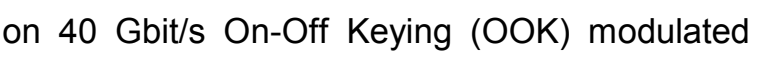

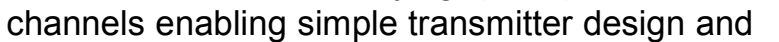

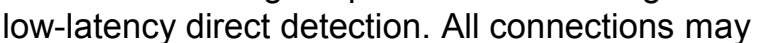

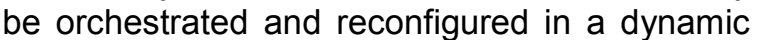

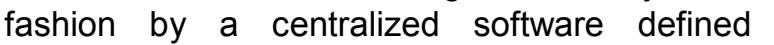

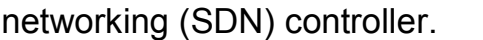

\section{Data centre network architecture}

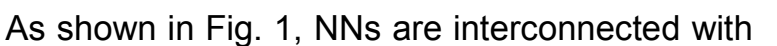

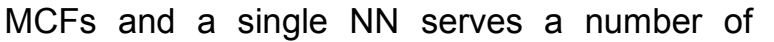

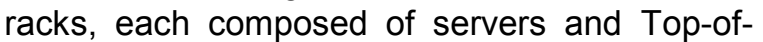

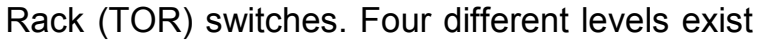

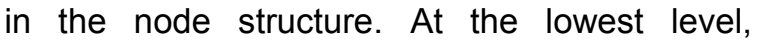

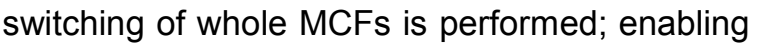

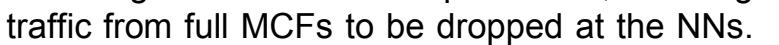

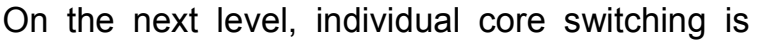

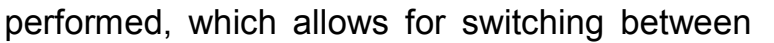

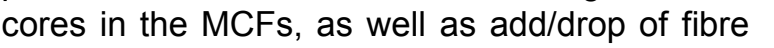

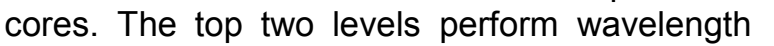

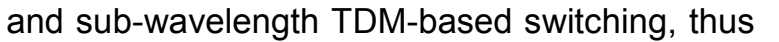

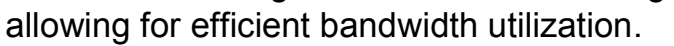

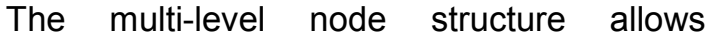

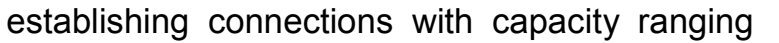

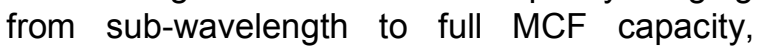

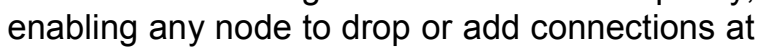

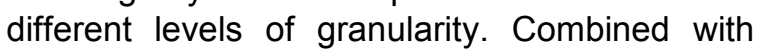

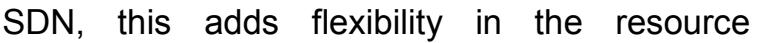

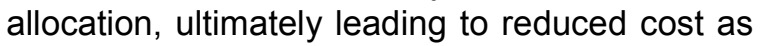

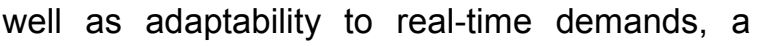

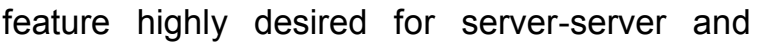

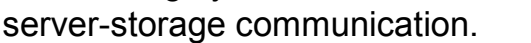

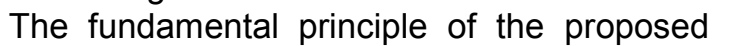

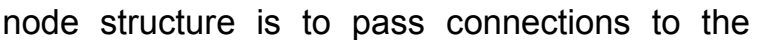

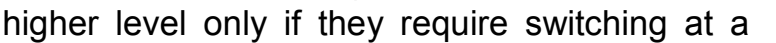

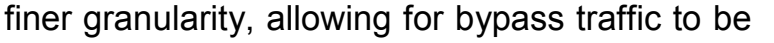

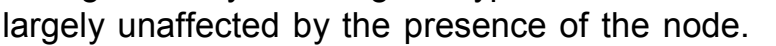

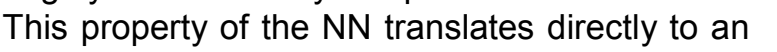

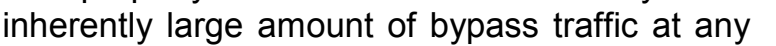

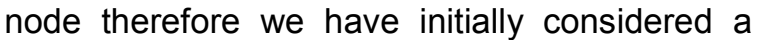

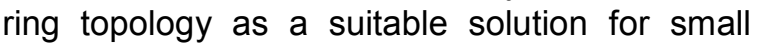

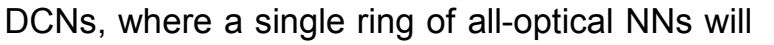

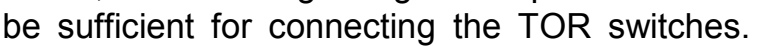

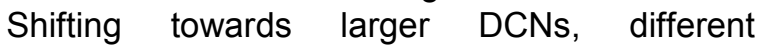

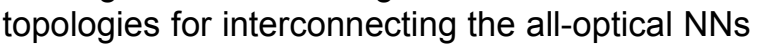
$\square$
$\mathrm{m}$ 


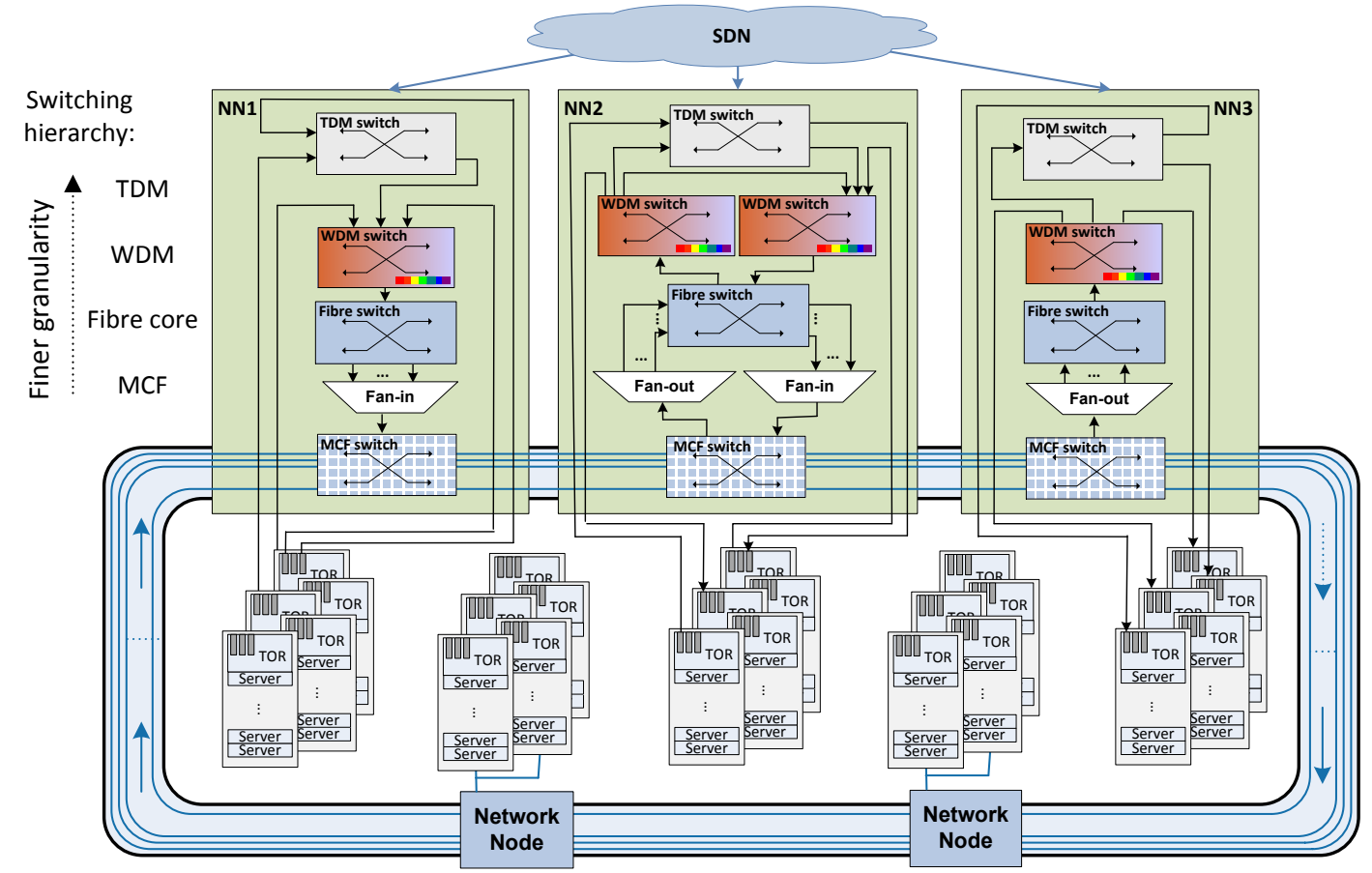

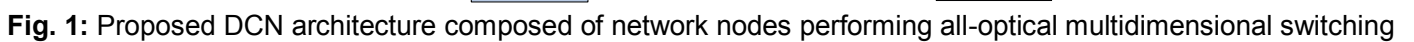

\section{Experimental setup and results}

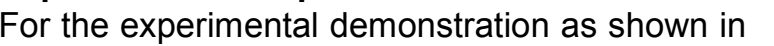

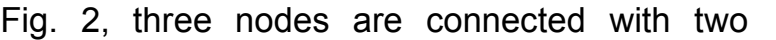

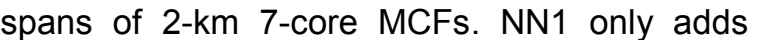

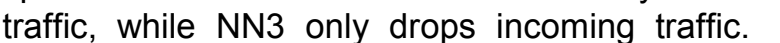

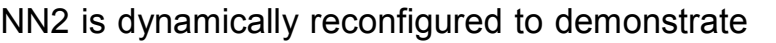

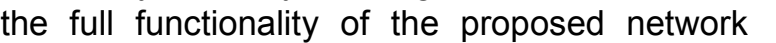

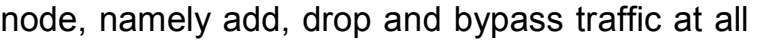

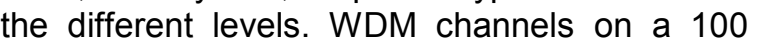

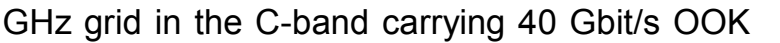

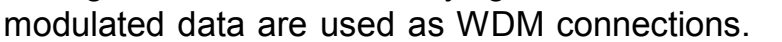

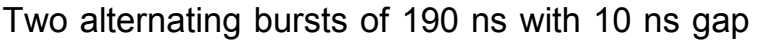

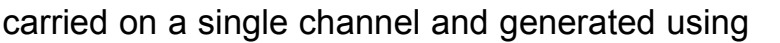

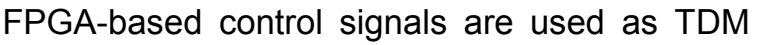

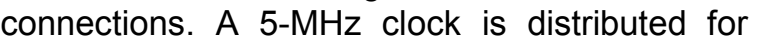

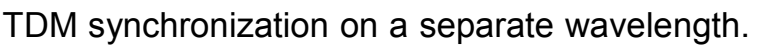

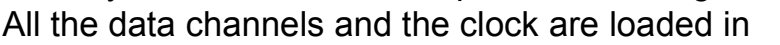

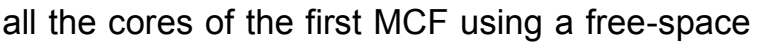
प

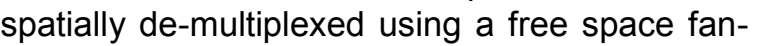

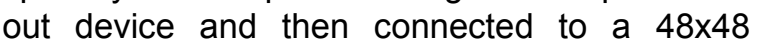

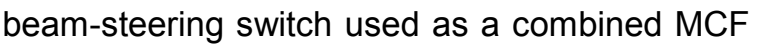

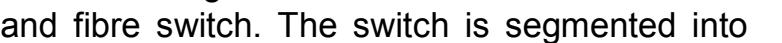

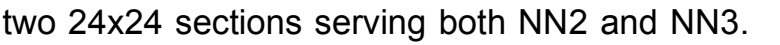

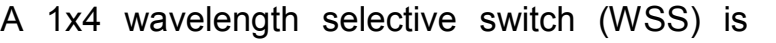

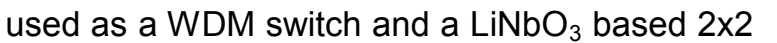
प

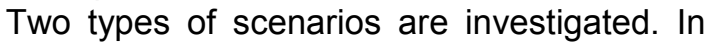
the case of 'full bypass', all connections from

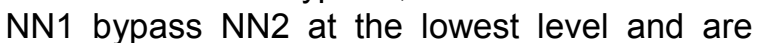

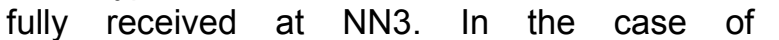
'add/drop/bypass', connections from NN1 target

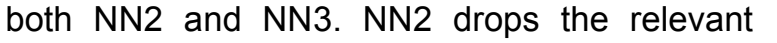

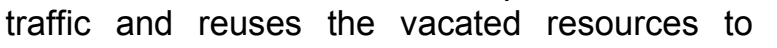

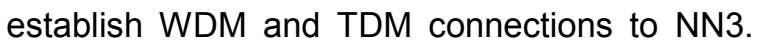

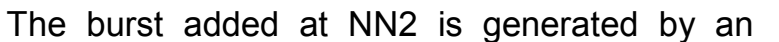

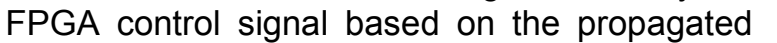

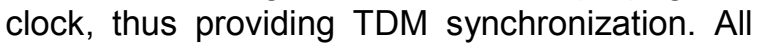

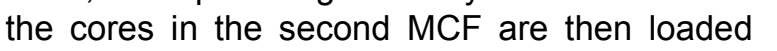

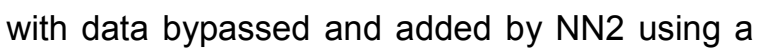
प

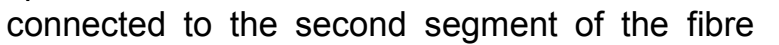

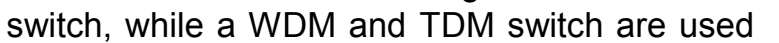

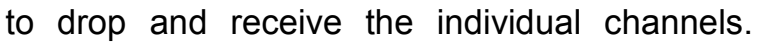

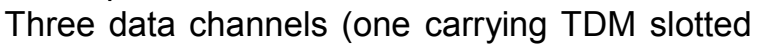

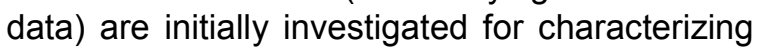

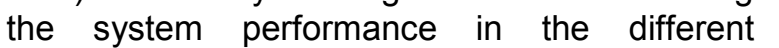

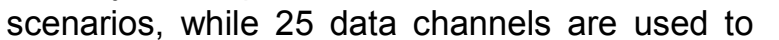

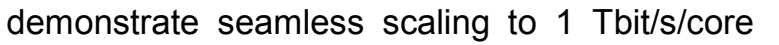

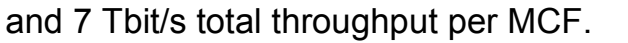

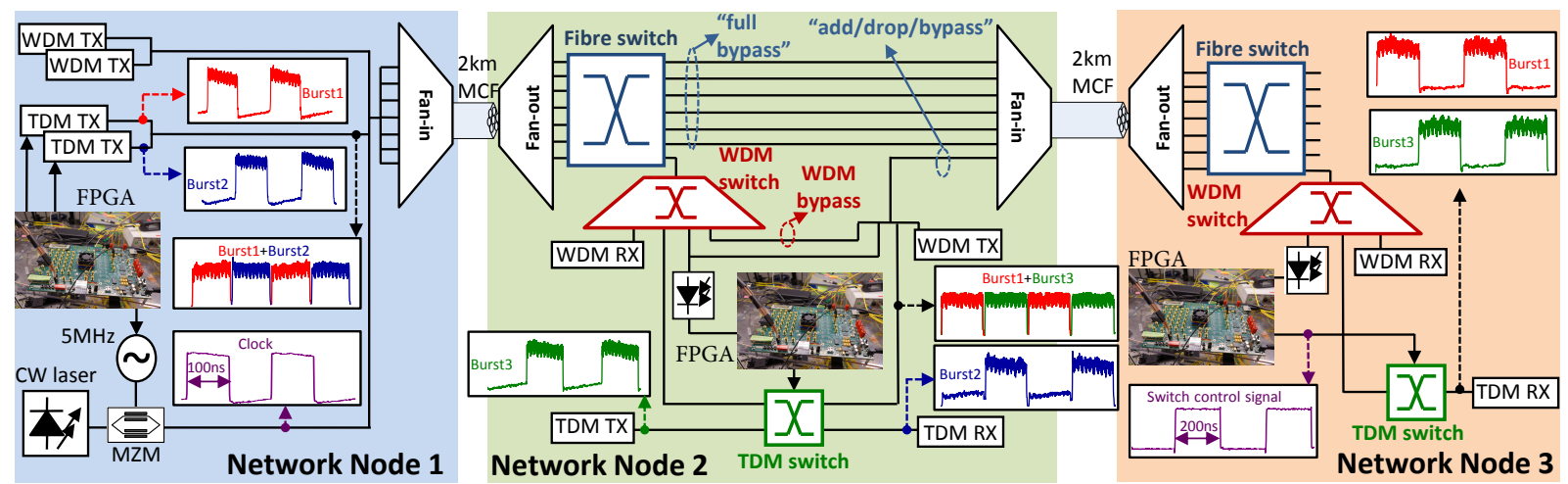

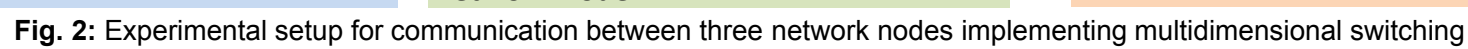



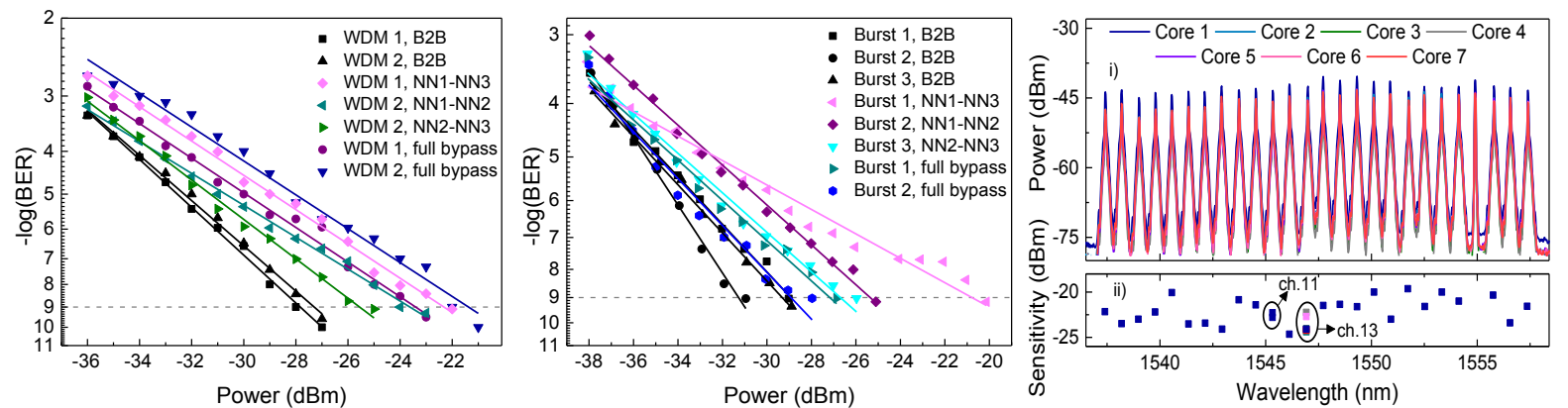

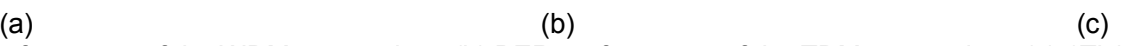

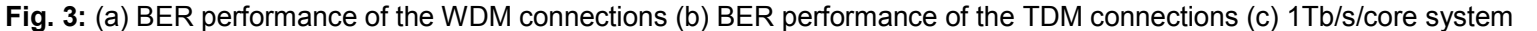

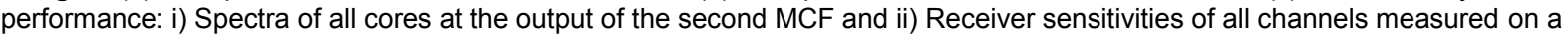

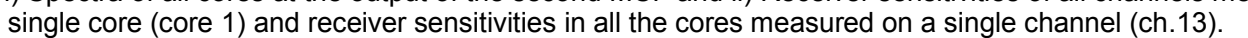

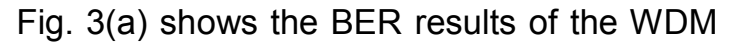
connections in the 'full bypass' and

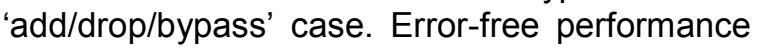
'

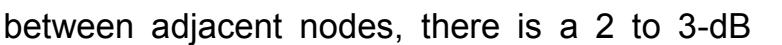

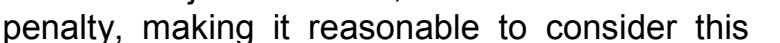
प प

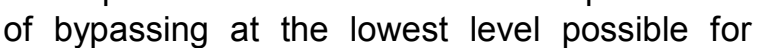

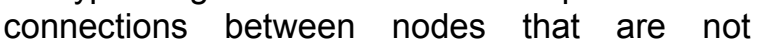

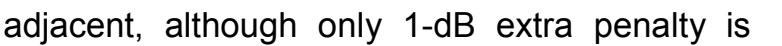

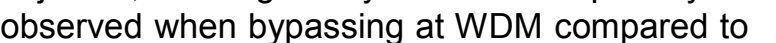

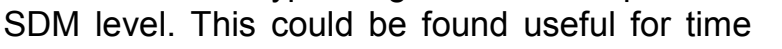

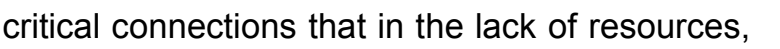

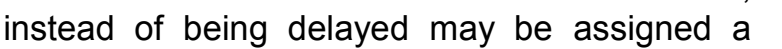

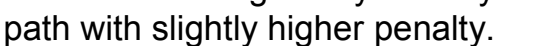

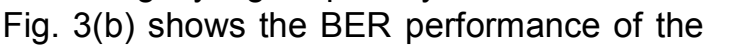
TDM connections in the 'full bypass' and 'add/drop/bypass' scenario. All connections '

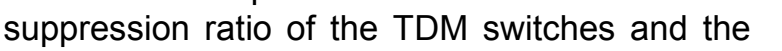

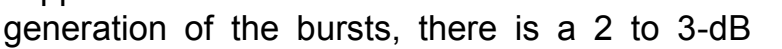

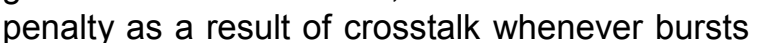

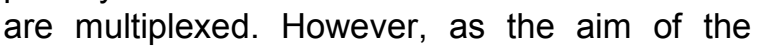

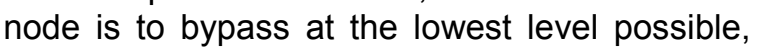

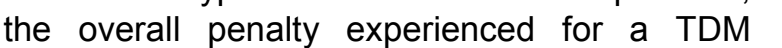

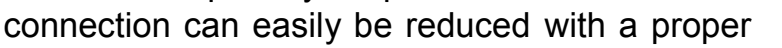

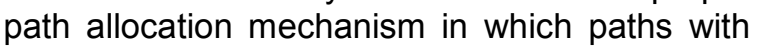

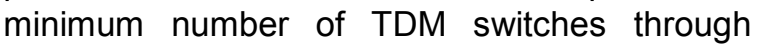

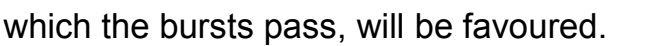

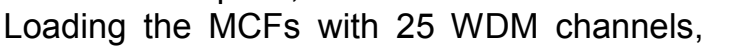

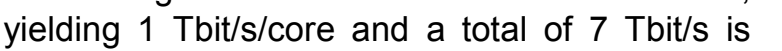

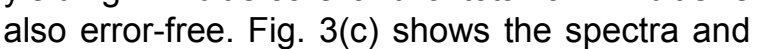

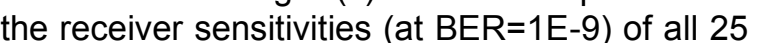
channels in the 'full bypass' scenario. The B2B

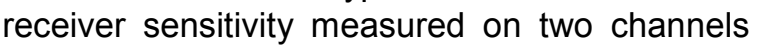

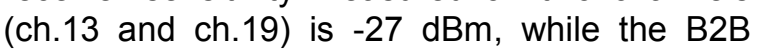

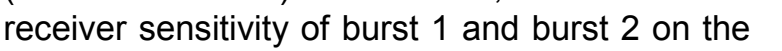

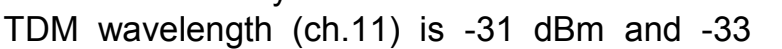

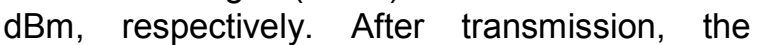

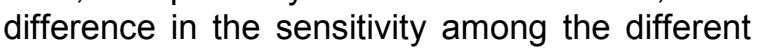

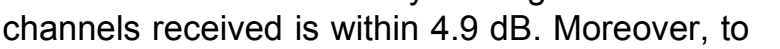

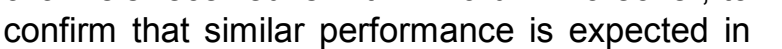

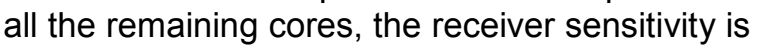

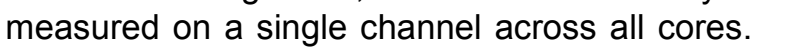

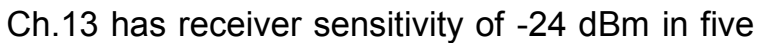

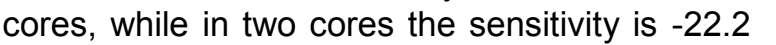

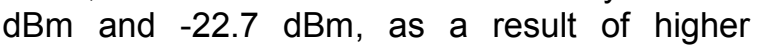

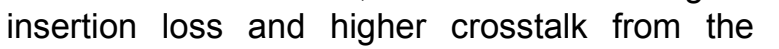

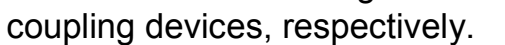

\section{Conclusions}

$\square$
0

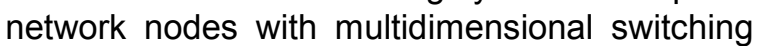

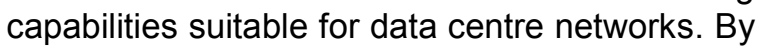

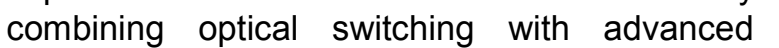

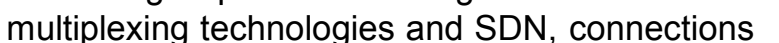

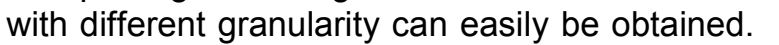

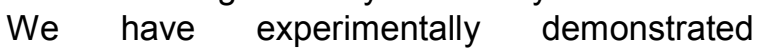

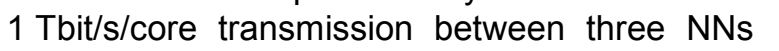

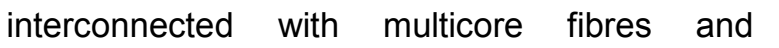

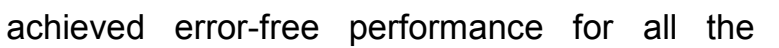
प

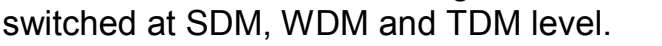

\section{Acknowledgements}

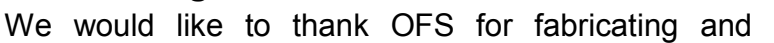

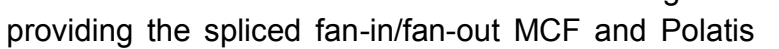
प

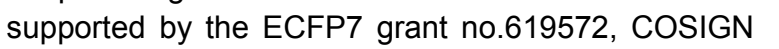

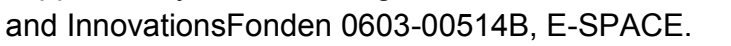

\section{References}

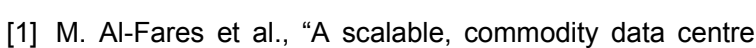

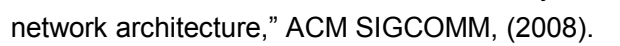

mIN. Farrington et al., "Helios: A hybrid electrical/optical switch architecture for modular data centers," ACM

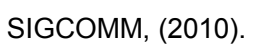

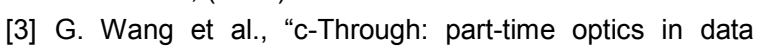
centers," ACM SIGCOMM,

पाuZ. Cao et al., "Experimental demonstration of $\square \square \square \square \square$

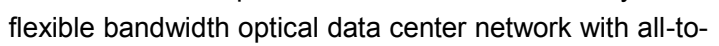

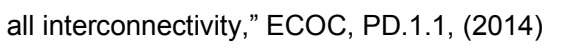

M. S. Tucker, "Scalability and energy consumption of optical and electronic packet switching," J. Lightw.

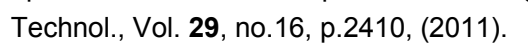

U. Yan et al., "First demonstration of all $\square \square \| m$

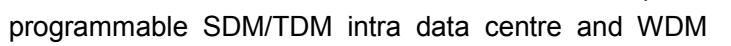

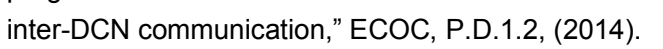

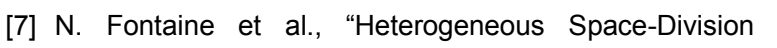

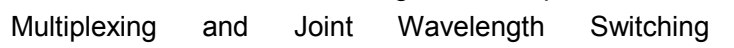

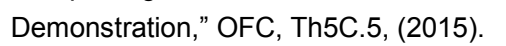

